\title{
Qualidade de vida e autoeficácia em atletas brasileiros de voleibol de rendimento
}

https://doi.org/10.11606/issn.1981-4690.v35i1p177-186

\section{Paulo Vitor Suto Aizava* Guilherme Moraes Balbim** J osé Roberto Andrade do Nascimento J unior ${ }^{* *+}$ Sidney Luciano Papke Lenamar Fiorese*}

\section{Resumo}

Este estudo investigou a associação entre a qualidade de vida (OV) e a autoeficácia de atletas de voleibol de alto rendimento. Participaram da pesquisa 86 atletas de nivel municipal, estadual e nacional. Como instrumentos foram utilizados a Escala de Auto Eficácia Geral Percebida e o Formulário Curto Para Pesquisa em Saúde (SF-36). Na análise dos dados, utilizou-se o teste de Kolmogorov Smirnov, KurskalWallis, Friedman e Qui-quadrado $(p<0,05)$. Os resultados evidenciaram que os atletas apresentaram boa $\mathrm{QV}$, entretanto, não houve diferença significativa em função do nível competitivo $(p>0,05)$. Ao comparar a QV intragrupos, o domínio que mais se destacou em todos os grupos foi a capacidade funcional $(p<0,05)$. Em relação à faixa etária, verificou-se que os atletas mais velhos apresentaram maior limitação por aspectos físicos em comparação aos adultos jovens $(\mathrm{P}<0,05)$, os quais por sua vez demonstraram menor QV em relação à dor em comparação com os jovens atletas $(p<0,05)$. Os domínios de Vitalidade, Aspectos sociais e Limitação por aspectos emocionais da QV apresentaram associação com o alto nível de autoeficácia $(p<0,05)$. Concluiu-se que o contexto de alto rendimento do voleibol brasileiro pode ser considerado um ambiente propulsor de QV para os atletas, além do que a autoeficácia é um elemento interveniente na $\mathrm{OV}$ de atletas de voleibol de alto rendimento.

Palavras-chave: Qualidade de vida; Auto eficácia; Esporte.

\section{Introdução}

É consenso na literatura que a prática de atividade física e esporte é um importante fator para a melhora da qualidade de vida $(\mathrm{QV})$ relacionada à saúde ${ }^{1,2}$. Tal reconhecimento se deve ao fato da prática esportiva promover no indivíduo a sensação de bem-estar, a manutenção da saúde física, emocional e de funções intelectuais, além de proporcionar diversas interaçôes sociais ${ }^{1,3}$.

Por um lado, pesquisas têm demonstrado a influência positiva da prática de atividade física regular na QV e nas relaçóes entre variáveis do funcionamento psicossocial de indivíduos não atletas ${ }^{1,4}$. Em contrapartida, investigaçóes na área das ciências do esporte ressaltam que a partir do momento que o esporte passa a ser de alto rendimento, a relação positiva entre a prática esportiva ea $Q V$ passa a ser questionável'5 .

Tal questionamento ocorre devido ao esporte de alto nível ser visto muitas vezes como um meio não saudável para o atleta alcançar a QV, principalmente devido a um cotidiano de intensos treinamentos
*Departamento de Educação Física, Universidade Estadual de Maringá, Maringá, PR, Brasil.

** Universidade Estadual do Oeste do Paraná, Marechal Cândido Rondon, PR, Brasil.

***Departamento d e E d u c a çã o Física, Faculdade Metropolitana de Maringá, Maringá, PR, Brasil.

${ }^{* * * *}$ Seleção Brasileira de Voleibol, C on feder ação Brasileira de Voleibol (CBV), Saquarema, RJ, Brasil. 
físicos e técnicos, bem como pressões advindas dos técnicos e torcida, possíveis desilusóes com o esporte e muitas vezes distanciamento da família e amigos $^{6}$. As lesôes que frequentemente ocorrem em qualquer nível competitivo, assim como a elevada exigência física nos treinamentos diários, afetando negativamente os aspectos físicos e psicológicos, e consequentemente a $\mathrm{QV}^{5}$.

Apesar de tais evidências, a nossa pesquisa bibliográfica aponta que não existem pesquisas sobre a QV no contexto esportivo de alto rendimento, além do que não há consenso na literatura a respeito dos benefícios do esporte de alto rendimento para a QV. A maioria das investigaçóes recentes sobre QV fazem referência à relação de exercícios físicos com fatores como terceira idade ${ }^{7}$, infância e adolescência ${ }^{1}$, obesidade $^{8}$, e uso de tabaco 9 . Assim, o presente estudo pretende explorar esta lacuna, investigando a $\mathrm{QV}$ no contexto esportivo.

Estudos recentes na área das ciências da saúde têm verificado que o bem-estar e comportamentos positivos relacionados à saúde e à $\mathrm{QV}$ possuem relação com níveis positivos de autoeficácia ${ }^{10}$. De acordo com a perspectiva sócio cognitiva de Albert Bandura, base teórica do presente estudo, a autoeficácia desempenha funçóes fundamentais no comportamento humano, tais como: metas e aspiraçóes, expectativas de resultados, tendências afetivas, percepçóes dos impedimentos (obstáculos) e oportunidades do meio social. Considerando que a autoeficácia caracteriza-se como um processo dinâmico do comportamento, a teoria sócio-cognitiva revela ser uma interessante base teórica para seu estudo, uma vez que explica o comportamento no contexto social, enfatizando os processos cognitivos do ser humano ${ }^{11}$.

Nessa perspectiva, pesquisadores têm observado que a

\section{Método}

Trata-se de um estudo descritivo correlacional transversal em que a populaçáo alvo deste estudo foi composta por atletas adultos de voleibol masculino. Foram convidados a participar da pesquisa todos os atletas adultos da seleçáo brasileira de voleibol masculino convocados para as competiçóes da temporada de 2010, as quatro equipes participantes do $3^{\circ}$ Grand Prix (GP) Estadual de Voleibol do Paraná - 2010 e as três equipes de voleibol melhor classificadas para os 53 Jogos Abertos do Paraná (JAP'S) - 2010.

Assim, fizeram parte da pesquisa 47 atletas de autoeficácia também pode ser um elemento psicológico interveniente para o contexto esportivo de alto rendimento, uma vez que pode exercer função auxiliar no processo ensino-aprendizagem, no gerenciamento $\mathrm{da}$ ansiedade ${ }^{12}$, estresse ${ }^{13}$ e consequentemente, na melhora no desempenho dos atletas ${ }^{14}$. A autoeficácia no contexto esportivo também tem sido associada com outras variáveis psicológicas, tais como: ansiedade ${ }^{15}$, motivação $^{16}$, agressáa ${ }^{17}$ e personalidade ${ }^{18}$. Apesar de a literatura apontar a importância da autoeficácia para a saúde e o bem-estar ${ }^{11}$, os quais estão diretamente relacionados com a $\mathrm{QV}$, não foram encontradas pesquisas associando tais variáveis em atletas de alto rendimento, revelando assim mais uma lacuna que o presente estudo pretende explorar.

Percebe-se assim, a relevância cientifica e social de pesquisas que relacionem a autoeficácia à $\mathrm{QV}$ no contexto esportivo, visto que a literatura aponta que a autoeficácia pode ser considerada um elemento interveniente para comportamentos positivos relacionados à saúde e bem-estar ${ }^{10}$, além de evitar os efeitos negativos de alguns transtornos psicológicos ${ }^{11}$. Tais relaçóes foram exploradas em indivíduos não-atletas por HuANG et al. ${ }^{19}$, revelando a necessidade de futuras investigaçóes no contexto esportivo. Dessa forma, os achados do presente estudo podem servir como novos parâmetros sobre as relaçóes entre tais variáveis no esporte de alto rendimento.

Face ao exposto, o presente estudo teve como objetivo analisar o nível de qualidade de vida e auto eficácia de atletas de voleibol de alto rendimento, buscando especificamente comparar a QV e a auto eficácia em função do nível de desempenho e faixa etária dos atletas, além de verificar a associação entre a QV e a auto eficácia. nível municipal participantes dos JAP'S - 2010, 26 atletas de nível de estadual participantes do GP e 13 atletas de nível nacional da seleção brasileira - 2010, totalizando 86 atletas de voleibol masculino adulto.

Utilizou-se uma ficha de identificação com fins de registro dos dados pessoais dos atletas com nome, data de nascimento, endereço, tempo de prática, idade de início, tempo de treinamento diário, dias de treinamento e conquista de medalhas.

Para a análise da autoeficácia utilizou-se a Escala de Auto Eficácia Geral Percebida (The General SelfEfficacy Scale), adaptada a validada para a língua 
portuguesa por Souza e Souza ${ }^{20}$. A escala foi criada para avaliar um senso geral de autoeficácia percebida, objetivando predizer a capacidade de superação das dificuldades diárias assim como a adaptação depois de experimentar todos os tipos de eventos estressantes da vida. $\mathrm{O}$ instrumento possui 10 itens sobre opinióes pessoais do indivíduo a respeito de si mesmo, os quais são respondidos por meio de uma escala Likert de 4 pontos num continuum de 1 (nem um pouco verdadeiro) até 4 (exatamente verdadeiro). A pontuação vem de 10 pontos (autoeficácia baixa) a 40 pontos (autoeficácia alta). Os resultados foram categorizados em: baixa (10 - 20 pontos), média (21 30 pontos) e alta autoeficácia (31 - 40 pontos). Neste estudo, a escala apresentou $\alpha=0,85$, demonstrando uma forte confiabilidade dos dados.

Para a análise da qualidade de vida, foi utilizado o Formulário Curto Para Pesquisa em Saúde (Medical Outcome Study 36 - Item Short - Form Health Survey) (SF-36), que foi criado a partir de uma revisão dos instrumentos ligados a qualidade de vida já existente na literatura nos últimos $20 \operatorname{anos}^{21}$. Este instrumento avalia 8 aspectos distintos: capacidade funcional; aspectos físicos; aspectos emocionais; dor; estado geral de saúde; vitalidade; aspectos sociais e saúde mental. As questóes possuem escala do tipo Likert de 7 pontos num continuum de 1 a 7 pontos. A pontuação final é de zero a 100 pontos, em que zero é considerado o pior estado de qualidade de vida e 100 o melhor estado de qualidade de vida. Neste sentido, para investigar associaçáo das variáveis foi necessário dividir os resultados deste questionário em três categorias: regular, boa e muito boa. Desta forma, os resultados foram categorizados em regular (0 - 30 pontos) boa (31 - 60 pontos) e muito boa qualidade de vida (61 - 100 pontos). No presente trabalho, este instrumento apresentou uma forte confiabilidade com $\alpha=0,76$.

Esta pesquisa foi aprovada pelo Comitê

\section{Resultados}

Nota-se que os atletas apresentaram muito boa qualidade de vida em todos os domínios (TABELA 1). A capacidade funcional foi o domínio com maior destaque, com 93\% ( $\mathrm{n}=80)$ dos atletas demonstrando muito boa qualidade de vida, seguindo dos aspectos sociais (82,6\% com muito boa QV) e limitação por aspectos emocionais (76,7\% com muito boa QV).
Permanente de Ética em Pesquisa Envolvendo Seres Humanos sob o parecer no 297/2010. Inicialmente, solicitou-se a autorização da Confederação Brasileira de Voleibol (CBV) para realização da coleta com os atletas da seleção brasileira (nível nacional), assim como para as equipes de nível municipal e estadual participantes do $3^{\circ}$ Grand Prix Estadual de Vôlei do Paraná e dos 53 Jogos Abertos do Paraná. Após a autorização da CBV e dos diretores e treinadores das equipes, foram marcadas datas para assinatura do Termo de Consentimento Livre e Esclarecido e para a coleta de dados. Os testes psicométricos foram aplicados em dias e horários disponibilizados pelos jogadores e pelos técnicos nos locais de treinamento das equipes durante a temporada 2010.

Para análise da distribuição dos dados foi utilizado o teste Kolmogorov-Smirnov, como os dados não apresentaram distribuição normal, foram utilizados Mediana (Md) e Quartis (Q1; Q3) para caracterização dos resultados dos atletas, além da frequência e percentual. Para a avaliação da consistência interna dos questionários de qualidade de vida e autoeficácia, foi utilizado o alfa de Cronbach. Para a comparação das dimensóes de qualidade de vida em função do nível de desempenho (municipal, estadual e nacional) e faixa etária (15-22, 23-30 e 31-38) dos atletas utilizou-se o teste de Kurskal-Wallis seguido do teste "U” de MannWhitney para comparação entre pares de grupos, visto que a normalidade e a homogeneidade dos dados foram violadas. Para verificar os domínios de qualidade de vida de maior destaque entre os atletas foi utilizado o Teste de Friedman seguido do teste de Wilcoxon para comparação entre pares de domínios. Para a associação entre a qualidade de vida e a auto eficácia foi utilizado o teste de Qui-quadrado, sendo que foi efetuado o 'V' de Cramer para identificar as medidas de efeito entre as associaçóes significativas, e o teste exato de Fisher quando necessário. Adotou-se significância de $\mathrm{P} \leq 0,05$.

Percebe-se que o domínio com menor prevalência de atletas com muito boa QV foi a dor (61,6\%).

Em relação à autoeficácia, houve prevalência de alto nível $(60,5 \%)$, enquanto que apenas um atleta $(1,1 \%)$ apresentou percepção baixa sobre a auto eficácia e 38,4\% demonstraram nível médio de autoeficácia. 
TABELA 1 - Frequência e percentual da qualidade devida dos atletas de voleibol masculino adulto.

\begin{tabular}{lccc}
\hline \multirow{2}{*}{ Qualidade de vida } & Regular & Boa & Muito boa \\
\cline { 2 - 4 } & $\%$ & $\%$ & $\%$ \\
\hline Capacidade funcional & 04,7 & 02,3 & 93,0 \\
Limitaçáo por aspectos físicos & 15,1 & 10,5 & 74,4 \\
Dor & 04,7 & 33,7 & 61,6 \\
Estado geral de saúde & 01,2 & 25,6 & 73,3 \\
Vitalidade & 02,3 & 30,2 & 67,4 \\
Aspectos sociais & 04,7 & 12,8 & 82,6 \\
Limitação por aspectos emocionais & 09,3 & 14,0 & 76,7 \\
Saúde mental & 01,2 & 24,4 & 74,4 \\
\hline
\end{tabular}

Verificou-se (TABELA 1) que em todos os níveis de desempenho os atletas possuem nível de boa (31 - 60 pontos) para muito boa qualidade de vida (61 - 100 pontos). Não foi encontrada diferença significativa ao comparar os domínios de QV em funçáo do nível de desempenho dos atletas. Ao comparar os domínios dentro de cada grupo, observou-se que a capacidade funcional dos atletas de nível municipal apresentou diferença significativa com os domínios de dor $(P=0,00)$, estado geral de saúde $(P=0,00)$, vitalidade
$(P=0,00)$ e saúde mental $(P=0,00)$, indicando que estes jogadores percebem melhor $\mathrm{QV}$ em relaçáo a capacidade funcional. Isso demonstra que estes atletas se sentem muito eficazes para realizar suas atividades diárias. Outra diferença encontrada foi dos aspectos sociais $(\mathrm{P}=0,03)$ em relaçáo à dor. A TABELA 2 apresenta a comparaçáo do nível de qualidade de vida em função do nível de desempenho dos atletas, e a comparaçáo entre os domínios da qualidade de vida em cada nível de desempenho.
Nota:

testes de Wilcoxon e Friedman foram utilizados para comparação dentro dos domínios em cada grupo.

CF: capacidade funcional,

LAF: limitação por aspectos físicos,

EGS: estado geral de saúde,

AS: aspectos sociais,

LAE: limitação por

aspectos emocionais,

SM: saúde mental.

*a: diferença da capacidade funcional em relação à dor, estado geral de saúde, vitalidade e saúde mental $(P \leq 0,01)$; *b: diferença dos aspectos sociais em relação à dor $(P \leq 0,05)$;

${ }^{*} \mathrm{c}$ : diferença da capacidade funcional em relação à dor, estado geral de saúde, vitalidade $(P \leq 0,05)$;

*d: diferença dos aspectos sociais em relação à vitalidade $(P \leq 0,05)$;

*e: diferença da saúde mental em relação à dor $(P \leq 0,05)$.

TABELA 2 - Mediana e primeiro e terceiro quartil dos níveis de qualidade de vida de acordo com o nível de desempenho dos atletas e valores de P do teste de Kruskal-Wallis.

\begin{tabular}{|c|c|c|c|c|}
\hline \multirow{2}{*}{ Qualidade de vida } & Municipal $(n=47)$ & Estadual $(\mathrm{n}=26)$ & Nacional $(n=13)$ & \multirow{2}{*}{$P$} \\
\hline & Md (Q1-Q3) & Md (Q1-Q3) & Md (Q1-Q3) & \\
\hline $\mathrm{CF}$ & $\mathbf{9 5 , 0}(90,0-100,0)^{a}$ & $\mathbf{9 7 , 5}(88,7-100,0)^{\mathrm{c}}$ & $\mathbf{9 0 , 0}(82,5-100,0)^{\mathrm{c}}$ & 0,99 \\
\hline LAF & $100,0(50,0-100,0)$ & $100,0(75,0-100,0)$ & $50,0(--100,0)$ & 0,81 \\
\hline Dor & $64,0(51,0-84,0)$ & $62,0(58,7-84,0)$ & $51,0(41,0-67,0)$ & 0,70 \\
\hline EGS & $67,0(57,0-80,0)$ & $72,0(60,7-80,0)$ & $75,0(62,5-83,5)$ & 0,54 \\
\hline Vitalidade & $70,0(60,0-75,0)$ & $70,0(63,7-81,2)$ & $70,0(67,5-80,0)$ & 0,52 \\
\hline AS & $\mathbf{8 7 , 5}(62,5-100,0)^{\mathrm{b}}$ & $\mathbf{8 7 , 5}(71,8-100,0)^{\mathrm{d}}$ & $87,5(75,0-100,0)$ & 0,72 \\
\hline LAE & $100,0(33,3-100,0)$ & $100,0(66,6-100,0)$ & $100,0(100,0-100,0)$ & 0,39 \\
\hline SM & $80,0(60,0-84,0)$ & $80,0(60,0-88,0)$ & $\mathbf{8 8 , 0}(72,0-92,0)^{\mathrm{e}}$ & 0,23 \\
\hline
\end{tabular}

Na TABELA 2 pode-se observar que os valores medianos da capacidade funcional dos atletas de nível municipal e estadual foram significativamente superiores $(\mathrm{P} \leq 0,01)$ aos do domínio da dor, estado geral de saúde e vitalidade, resultado semelhante aos dos atletas de nível municipal. Os aspectos sociais também apresentaram maiores valores $(\mathrm{P} \leq 0,05)$ em comparação com a vitalidade. Os resultados dos atletas de nível nacional vão ao encontro dos achados dos outros dois grupos, 
visto que a capacidade funcional apresentou diferença significativa $(\mathrm{P} \leq 0,01)$ com os domínios de dor, estado geral de saúde e vitalidade. Outro domínio que se destacou foi à saúde mental em detrimento à dor $(\mathrm{P} \leq 0,05)$. A TABELA 3 apresenta a comparação do nível de qualidade de vida em função da faixa etária dos atletas.

Houve diferença significativa no domínio limitação por aspectos físicos entre os atletas de 23-30 anos em relação aos atletas de 31- 38 anos $(\mathrm{P} \leq 0,01)$, indicando que os adultos jovens percebem melhor QV neste fator. Em relação ao domínio de dor, houve diferença significativa entre os atletas jovens (15-22 anos) em comparação aos atletas de 23-30 anos $(\mathrm{P} \leq 0,05)$, evidenciando que os atletas mais jovens percebem melhor QV neste domínio (TABELA 3).

A TABELA 4 apresenta a associação da autoeficácia com o nível de desempenho dos atletas (municipal, estadual e nacional), faixa etária (15-22 anos, 23-30 anos, 31-38 anos), tempo de prática (1-7 anos, 8-15 anos, 16-24 anos) e qualidade de vida dos atletas de voleibol de rendimento $(\mathrm{n}=86)$.

Não houve associação da autoeficácia com o nível de desempenho dos atletas. No entanto, observou-se que a autoeficácia apresentou associação com a faixa etária $(P=0,04)$ e com o tempo de prática $(P=0,05)$, indicando que ser um atleta jovem (15-22 anos) e com pouca experiência profissional (1-7 anos) possuem um efeito de aproximadamente $15 \%$ no nível de autoeficácia.

$\mathrm{Na}$ associação da QV com a autoeficácia, foram encontradas associaçóes significativas nos domínios de vitalidade $(P=0,05)$, aspectos sociais $(P=0,03)$ e limitação por aspectos emocionais $(P=0,01)$, indicando associação entre alto nível de autoeficácia e muito boa QV. Nos aspectos sociais observou-se um efeito significativo $(V=0,35 ; P=0,03)$, assim como na limitação por aspectos físicos $(V=0,31 ; P=0,02)$. Tal resultado indica que os aspectos sociais positivos têm um impacto de aproximadamente $12 \%$ para uma alta autoeficácia, enquanto que a medida de efeito para limitaçóes físicas foi de aproximadamente $10 \%$.

TABELA 3 - Mediana e primeiro e terceiro quartil dos níveis de qualidade de vida de acordo com os 3 grupos de faixa etária e valores de P dos testes de Kruskal-Wallis e “U” de Mann-Whitney.

\begin{tabular}{|c|c|c|c|c|}
\hline \multirow{2}{*}{ Qualidade de vida } & $\begin{array}{l}15 \text { - } 22 \text { anos } \\
\text { (Jovens) }\end{array}$ & $\begin{array}{c}23-30 \text { anos } \\
\text { (Jovens Adultos) }\end{array}$ & $\begin{array}{l}31-38 \text { anos } \\
\text { (Adultos) }\end{array}$ & \multirow[t]{2}{*}{$P$} \\
\hline & Md (Q1-Q3) & Md (Q1-Q3) & Md (Q1-Q3) & \\
\hline $\mathrm{CF}$ & $95,0(90,0-100,0)$ & $95,0(90,0-95,0)$ & $95,0(62,5-100,0)$ & 0,06 \\
\hline LAF & $100,0(75,0-100,0)$ & $100,0(75,0-100,0)$ & $50,0(25,0-100,0)$ & $\mathbf{0 , 0 1 *}$ \\
\hline Dor & $72,0(52,0-84,0)$ & $51,1(41,0-72,0)$ & $61,0(41,0-84,0)$ & $\mathbf{0 , 0 3}{ }^{* *}$ \\
\hline EGS & $70,0(60,0-80,0)$ & $72,0(57,0-80,0)$ & $67,0(58,5-82,5)$ & 0,80 \\
\hline Vitalidade & $70,0(60,0-80,0)$ & $70,0(65,0-75,0)$ & $75,0(57,5-85,0)$ & 0,94 \\
\hline AS & $87,5(62,5-100,0)$ & $87,5(62,5-100,0)$ & $75,0(50,0-93,7)$ & 0,55 \\
\hline LAE & $100,0(66,6-100,0)$ & $100,0(33,3-100,0)$ & $100,0(66,7-100,0)$ & 0,88 \\
\hline SM & $80,0(64,0-88,0)$ & $84,0(68,0-89,0)$ & $64,0(58,0-92,0)$ & 0,47 \\
\hline
\end{tabular}

Nota:

CF: capacidade funcional, LAF: limitação por aspectos físicos, EGS: estado geral de saúde, AS: aspectos sociais, LAE: limitação por aspectos emocionais, SM : saúde mental. ${ }^{*}$ Diferença da categoria 23 - 30 anos em relação à categoria 31 - 38 anos $(P \leq 0,01)$;

**Diferença da categoria 15 - 22 anos em relação à categoria 23 - 30 anos $(P \leq 0,05)$. 
TABELA 4 - Associação da autoeficácia com o nível de desempenho, faixa etária, tempo de prática e QV dos atletas de voleibol.

*Associação significativa. Teste exato de Fisher $(P \leq 0,05)$;

${ }^{* *}$ Correlação significativa $(P \leq 0,05)$

\begin{tabular}{|c|c|c|c|c|c|c|c|c|}
\hline \multirow{3}{*}{ Variáveis } & \multicolumn{5}{|c|}{ Autoeficácia } & \multirow{3}{*}{$X^{2}$} & \multirow{3}{*}{$P$} & \multirow{3}{*}{$V$} \\
\hline & $\begin{array}{l}\text { Média } \\
(n=34)\end{array}$ & & & & & & & \\
\hline & $\%$ & $f$ & $\%$ & $f$ & $\%$ & & & \\
\hline
\end{tabular}

\section{Nível de desempenho}

\begin{tabular}{|c|c|c|c|c|c|c|c|c|c|}
\hline Municipal & 21 & 44,7 & 26 & 55,3 & 47 & 100 & & & \\
\hline Estadual & 10 & 38,5 & 16 & 61,5 & 26 & 100 & 2,63 & 0,17 & - \\
\hline Nacional & 3 & 23,1 & 10 & 76,9 & 13 & 100 & & & \\
\hline \multicolumn{10}{|l|}{ Faixa etária } \\
\hline $15-22$ anos & 22 & 46,8 & 25 & 53,2 & 47 & 100 & & & \\
\hline $23-30$ anos & 10 & 38,5 & 16 & 61,5 & 26 & 100 & 4,77 & $0,04^{*}$ & 0,16 \\
\hline $31-38$ anos & 2 & 15,4 & 11 & 84,6 & 13 & 100 & & & \\
\hline \multicolumn{10}{|l|}{ Tempo de Prática } \\
\hline $1-7$ anos & 18 & 50,0 & 18 & 50,0 & 36 & 100 & & & \\
\hline $8-15$ anos & 13 & 35,1 & 24 & 64,9 & 37 & 100 & 4,37 & $0,05^{*}$ & 0,15 \\
\hline $16-24$ anos & 3 & 23,1 & 10 & 76,9 & 13 & 100 & & & \\
\hline \multicolumn{10}{|l|}{ Qualidade de Vida } \\
\hline Capacidade funcional & 32 & 40,1 & 48 & 60,0 & 80 & 100 & 1,57 & 0,94 & - \\
\hline Limitação por aspectos físicos & 23 & 36,0 & 41 & 64,1 & 64 & 100 & 2,12 & 0,43 & - \\
\hline Dor & 19 & 35,8 & 34 & 64,2 & 53 & 100 & 3,18 & 0,48 & - \\
\hline Estado geral de saúde & 25 & 39,7 & 38 & 60,3 & 63 & 100 & 2,02 & 0,91 & - \\
\hline Vitalidade & 19 & 32,8 & 39 & 67,2 & 58 & 100 & 5,12 & $0,05^{*}$ & 0,17 \\
\hline Aspectos sociais & 26 & 36,6 & 45 & 63,4 & 71 & 100 & 21,83 & $\mathbf{0 , 0 3 *}$ & $0,35^{* *}$ \\
\hline $\begin{array}{l}\text { Limitação por aspectos } \\
\text { emocionais }\end{array}$ & 20 & 30,3 & 46 & 69,7 & 66 & 100 & 16,64 & $0,01^{*}$ & $0,31^{* *}$ \\
\hline Saúde Mental & 24 & 37,5 & 40 & 62,5 & 64 & 100 & 4,78 & 0,24 & - \\
\hline
\end{tabular}

\section{Discussão}

Até onde se sabe, esse é o primeiro estudo a investigar a associação entre a QV e a autoeficácia de atletas de voleibol de diferentes níveis de desempenho (municipal, estadual e nacional), evidenciando a relevância dos achados desta pesquisa para o contexto esportivo. Nossos resultados sugerem que atletas com alto nível de autoeficácia possuem maiores chances de apresentaram melhor $\mathrm{QV}$, principalmente em relaçáo aos aspectos emocionais, sociais e de vitalidade (TABELA 4). Ressalta-se também que para os atletas de voleibol da presente pesquisa, o esporte de alto rendimento está relacionado com bom nível de QV (TABELA 1). Tal achado pode ser considerado um novo indicador na relaçáo entre esporte de alto rendimento e $\mathrm{QV}$, visto que pesquisas recentes têm apontado que a partir do momento que o esporte passa a ser de alto rendimento, a QV do atleta sofre uma queda significativa ${ }^{6}$.

A associação entre a QV e o alto nível de autoeficácia encontrada no presente estudo (TABELA 4) apresenta suporte em alguns princípios da teoria sócio cognitiva de BANDURA ${ }^{11}$. Tal teoria aponta que a autoeficácia 
entendida como o julgamento do indivíduo sobre as próprias capacidades para organizar e executar cursos de ação exigidos para atingir certo desempenho, tem função reguladora sobre o comportamento da pessoa, colaborando assim para a qualidade do funcionamento psicossocial. Assim, percebe-se que como observados nossos achados, as crenças de autoeficácia podem levar a boa percepção de QV, visto que esta é entendida como a percepção individual da vida no contexto sociocultural em relação à suas metas, expectativas, padróes e preocupaçóes ${ }^{22}$.

Em relação a faixa etária e tempo de prática, observou-se que quanto mais velho e experiente o jogador (TABELA 4), maior a prevalência de alta autoeficácia. Tal achado encontra suporte no estudo de STEFANELLO ${ }^{23}$, a qual aponta que a autoeficácia por não ser um traço estável de personalidade, e sim um processo dinâmico, pode ser elevada ou diminuída ao longo da vida. Estes resultados ressaltam as explicaçóes de BANDURA, AzZi e POLYDORO ${ }^{11}$ sobre o comportamento humano e a interação recíproca de fatores pessoais (cogniçóes e afetos), ambientais e comportamentais, determinando assim a reciprocidade contínua para o estabelecimento da autoeficácia.

Percebeu-se que o alto nível de autoeficácia associase aos aspectos de QV de vitalidade, aspectos sociais e limitaçóes por aspectos emocionais (TABELA 4). Esses resultados podem estar relacionados com os processos cognitivos, motivacionais, afetivos e de seleção que constituem a autoeficácia ${ }^{11}$. A vitalidade (altos níveis de ativação para realização de tarefas) e os aspectos sociais da QV podem ser associados com os processos motivacionais da autoeficácia, os quais são compreendidos como açóes intencionais e dirigidas a uma meta e que dependem da interação de fatores intrínsecos e extrínsecos ${ }^{24}$. A alta QV no que se refere aos aspectos emocionais pode-se relacionar com os processos afetivos, já que são reaçóes emocionais dos indivíduos experimentados em situações ameaçadoras, tais como o estresse e a ansiedade ${ }^{7}$.

Especificamente, a associação da autoeficácia com os aspectos sociais e a limitação por aspectos físicos evidencia que os altos níveis destas três variáveis estão positivamente e diretamente associados. Nesse sentido, Feltz, Short e Sullivan ${ }^{14}$ apontaram que quando ocorre desenvolvimento das atividades sociais sem interferência induzida por problemas físicos ou emocionais, consequentemente, o julgamento de valor sobre o que cada um pode fazer com suas próprias habilidades será alto.

$\mathrm{Na}$ identificação do nível de QV (TABELA 1), a maioria dos atletas evidenciou bom a muito bom nível de QV em todos os domínios. Este resultado vai de encontro com alguns achados ${ }^{25}$ com atletas profissionais chineses, no qual a QV nas dimensóes de limitação por aspectos físicos, dor, vitalidade e limitação por aspectos emocionais apresentou valores inferiores aos do presente trabalho. Identificaram ainda que os atletas profissionais apresentaram menor $\mathrm{QV}$ do que os amadores, ao contrário do presente estudo, no qual não foram evidenciadas diferenças significativas nas dimensóes de QV em função do nível competitivo dos atletas. Contudo, é importante salientar que na percepção da QV os aspectos subjetivos e os estímulos recebidos passam por processos subjetivos de avaliação, interpretação, percepção e assimilação das informaçóes ${ }^{3}$. Assim, nesta pesquisa percebe-se que tais percepçóes se encontraram semelhantes quando comparados os fatores que interferem na QV em todos níveis de desempenho.

Outro resultado interessante está relacionado à comparação do nível de QV em função da faixa etária dos atletas (TABELA 3). Observou-se que os atletas mais experientes (31-38 anos) são mais limitados fisicamente em comparação aos adultos jovens (23-30 anos), indicando que atletas em fim de carreira e com idade mais avançada estão mais suscetíveis às lesões. Tal resultado pode estar relacionado ao fato destes jogadores terem passado inúmeras temporadas sendo submetidos a cargas intensas de treinamentos e competições, além das próprias especificidades fisiológicas que ocorrem com o processo de envelhecimento ${ }^{21}$.

Além disso, verificou-se que os atletas entre 23 e 30 anos apresentaram mais dores em detrimento aos jovens atletas (15-22 anos), indicando que jogadores esta é uma faixa etária crítica no esporte de alto rendimento, uma vez que é a fase da carreira na qual os atletas alcançam o auge do desempenho esportivo e, consequentemente, são submetidos às maiores cargas de treinamento ${ }^{26}$.

Outro achado a ser ressaltado são os níveis de QV dos jovens atletas (15-22 anos). Os achados do presente estudo vão ao encontro da QV de atletas entre 17 e 23 anos da NCAA dos Estados Unidos, que demonstraram altos escores em todas as dimensóes ${ }^{27}$. Em contrapartida, são muito superiores aos de atletas colegiais norte-americanos de diversas modalidades esportivas, que apresentaram valores moderados em todos os domínios ${ }^{28}$. Tal diferença pode estar relacionada ao fato do esporte colegial e universitário Americano ser mais competitivo e possuir maiores cargas de treinamento em comparação ao brasileiro.

Em relação à prevalência de autoeficácia, os achados desta pesquisa apontam que, independente do atleta 
ser de nível internacional, nacional ou municipal, o esporte age como um meio propulsor de autoeficácia, indicando que o contexto esportivo é um ambiente positivo para o desenvolvimento desta característica psicológica $^{23}$. Estes resultados corroboram com diversos estudos que identificaram elevados níveis de autoeficácia em atletas de diversas modalidades Além disso, recentes investigaçóes têm destacado destacam a importância da autoeficácia para o sucesso e melhora do desempenho esportivo e a existência de uma relação mútua entre a saúde mental e o esporte ${ }^{29}$.

Outro aspecto de destaque é o fato de que a dor apresentou prevalência de boa e muito boa em 93,5\% dos atletas (TABELA 1), indicando que a dor causada pelas intensas cargas de treino e lesôes não influencia negativamente na percepção de QV. Para Silva, Rabelo e RuBio ${ }^{30}$, a dor é presença constante na vida de atletas de alto nível, o que pode resultar em problemas como constante convivência com lesóes e até encurtamento da vida profissional. Todavia, tanto no presente estudo, quanto no de Silva, Rabelo e Rubio ${ }^{30}$ a dor náo parece influenciar negativamente a saúde e QV dos atletas. Por outro lado, MoreIra et al. ${ }^{5}$ em uma revisão sistemática sobre lesóes e QV, apontam evidências de que lesóes muitas vezes causadas pela dor decorrente das altas cargas de treinamentos levam à diminuição da QV.

Dada à importância destas variáveis, este estudo evidenciou que o contexto esportivo de alto rendimento pode ser considerado um ambiente propulsor para a obtenção e manutenção de bons níveis de autoeficácia e QV. É importante salientar que nossos achados apresentam algumas limitações, como o não acompanhamento dos atletas e a utilização apenas de dados quantitativos, que nos mostram indicadores dos comportamentos observados. Além disso, os atletas de nível municipal e estadual representavam apenas um estado brasileiro, impossibilitando a generalização dos resultados, entretanto, estes jogadores eram oriundos de diferentes regióes do país.

Dessa forma, é necessário cuidado quanto à generalização dos resultados encontrados no presente estudo. Assim, sugere-se que futuras pesquisas adotem delineamentos longitudinais, possibilitando assim identificar o processo do desenvolvimento e aprimoramento destas variáveis psicológicas ao longo da carreira do atleta. É importante também a realização de estudos em outras modalidades esportivas e com atletas do sexo feminino, para que futuras comparaçóes possam ser realizadas.

Como implicaçóes práticas acredita-se que este estudo possa fornecer novos parâmetros para profissionais do contexto esportivo de alto rendimento subsidiarem a relação positiva da QV com o esporte, principalmente pelo fato de outras pesquisas e alguns meios de comunicação propagarem a ideia da relação negativa entre a QV e o esporte de alto rendimento. Dessa forma, os profissionais da área podem se utilizar do presente estudo como um indicativo de que esta ideia pode estar equivocada, desde que seja levada em consideração a subjetividade da QV e o contexto esportivo.

Finalizando, destaca-se que a investigação da QV é de suma importância no meio esportivo, na medida em que demonstrou ser fator interveniente no desempenho dos atletas, mesmo com as pressóes e o estresse presente ao longo da carreira esportiva. Assim, investigaçóes como esta demonstram os indicativos de reais benefícios que o esporte de rendimento pode proporcionar aos atletas de voleibol. 


\begin{abstract}
Quality of life and self-efficacy in Brazilian athletes of high performance volleyball

This study investigated the association between life quality (LO) and self-efficacy among high-performance volleyball athletes. Participants were 86 athletes from local, state and national level. The instruments used were the General Perceived Self-Efficacy Scale and the Short Form for Health Research (SF-36). For data analysis, it was used the Kolmogorov-Smirno, Kurskal-Wallis, Friedman and Chi-Square tests $(p<0,05)$. The results showed that athletes had good $L Q$, however, there was no significant difference according to the competitive level $(p>0,05)$. When compared the intra-group $L Q$, the domain that stood out in all groups was the functional capacity $(p<0,05)$. In terms of age, it was found that older athletes showed greater limitations due to physical aspects compared to young adults $(p<0,05)$, which in turn demonstrated lower $L Q$ related to pain compared to youth athletes $(p<0,05)$. The domains of vitality, social aspects and limitation by emotional aspects were associated with the high level of self-efficacy $(p<0.05)$. It was concluded that Brazilian volleyball high performance context can be considered a propellant environment of $L Q$ for the athletes. In addition, self-efficacy is an intervening element on the LQ among high performance volleyball athletes.
\end{abstract}

KEYWoRDs: Life quality; Self-efficacy; Sport.

\title{
Referências
}

1. Spengler S, Woll A. The more physically active, the healthier? The relationship between physical activity and health-related quality of life in adolescents: the MoMo study. J Phys Act Health. 2013;10:708-715.

2. Iulia-Doru T, Maria T. Leisure sports activities impact on adults personal development and quality of life. Procedia Soc Behav Sci. 2013;84:1090-1094.

3. Kenneth CL, Alison RSV, Bay RC, McLeod TCV. A unique patient population? Health-related quality of life in adolescent athletes versus general, healthy adolescent individuals. J Athl Train. 2013;48(2):233-241.

4. Gaskin CJ, Morris T. Physical activity, health-related quality of life, and psychosocial functioning of adults with cerebal palsy. J Phys Act Health. 2008;5(1):146-157.

5. Moreira NB, Vagetti GC, Oliveira V, Campos W. Association between injury and quality of life in athletes: a systematic review, 1980-2013. Apunts Med. Esport. 2014;49:123-38.

6. Viana DFW, Mezzaroba C. O esporte de rendimento faz mal à saúde? Uma análise das atletas da seleção brasileira de ginástica rítmica. Motriviv. 2013;25(41):190-205.

7. Porto DB, Guedes DP, Fernandes RA, Reichert FF. Perceived quality of life and physical activity in Brazilian older adults. Motri. 2012;8:33-41.

8. Heath GW, Brown DW. Recommended levels of physical activity and health related quality of life among overweight and obese adults in the united states, 2005. J Phys Act Health. 2009; 6: 403-411.

9. Amela D, Aalen D, Llvana H, Esad P. Relationship between quality of lifeand physical activities in relation to the tobacco smoking habits. Homo Sporticus. 2012;14:11-14.

10. Ashford S, Edmunds J, French DP. What is the best way to change self-efficacy to promote lifestyle and reacreational physical activity? A systematic review with meta-analysis. Br J Health Psychol. 2010;15:265-288.

11. Bandura A, Azzi RG, Polydoro SAJ. Teoria Sócio Cognitiva: conceitos básicos. São Paulo: Artmed; 2008.

12. De Pero R, Mingati C, Pesce C, Capranica L, Piacentini MF. The relationships between pre-competitive anxiety self-efficacy and fear of injury in elite team gym athletes. Kinesiology. 2013;45(1):63-72.

13. Tayama J, Yamasaki H, Tamai M, Hayashida M, Shirabe S, Nishiura K, et al. Effect of base-line self-efficacy on physical activity and psychological stress after a one-week pedometer intervention. Percept Mot Skills. 2102;114(2):407-418.

14. Feltz DL, Short SE, Sullivan PJ. Self-efficacy theory and application in sport. Champaign, IL: Human Kinetics; 2008.

15. Vieira LF, Teixeira CL, Vieira JLL, Filho AO. Autoeficácia e nível de ansiedade em atletas jovens do atletismo paranaense. Rev Bras Cineantropom Desempenho Hum. 2011;13(3):183-188. 
16. Barkoukis V, Koidou E, Tsorbatzoudis H. Effects of a motivational climate intervention on state anxiety, self-efficacy, and skill development in physical education. Eur J Sport Sci. 2010;10(3):167-177.

17. Willemse M, Smith MR, Van Wyk SB. The relationship between self-efficacy and aggression in a group of adolescents in the peri-urban town of Worcester, South Africa: Implications for sport participation. Afr J Phys Health Educ Recreat Dance. 2011;(Suppl):90-102.

18. Merritt CJ, Tharp IJ. Personality, self-efficacy and risk-taking in parkour (free-running). J Sport Exerc Psychol. 2013; 14: 608-611.

19. Huang L, Li L, Zhang Y, Li H, Li X, Wang H. Self-efficacy, medication adherence, and quality of life among people living with HIV in Hunan Province of China: a questionnaire survey. J Assoc Nurses AIDS Care. 2013;24:145-153.

20. Souza I, Souza MA. Validação da escala de autoeficácia geral percebida. Revista Univ Rural Série Ciências Humanas. 2004;26(1-2):12-17.

21. Ciconelli RM, Ferraz MB, Santos W, Meinão I, Quaresma MR. Tradução para a língua portuguesa e validação do questionário genérico de avaliação de qualidade de vida SF-36 (Brasil SF-36). Rev Bras Reumatol. 1999;39(3):143150.

22. Roeder MA. Atividade física, Saúde mental e qualidade de vida. Rio de Janeiro: Shape; 2003.

23. Stefanello JMF. Treinamento de competências psicológicas: em busca da excelência esportiva. Barueri: Editora Manole; 2007.

24. Gomes SS, Miranda R; Bara Filho MG; Bandão MRF. O fluxo no voleibol: relação com a motivaçáo, autoeficácia, habilidade percebida e orientação às metas. Rev Educ Fís UEM. 2012;23(3):379-387.

25. Cheung, RTH; Zhang, Z; Ngai, SPC. Different relationships between the Level of patellofemoral pain and quality of life in professional and amateur athletes. PMR. 2013;5(7):568-572.

26. Noce F, Costa VT, Simim MAM, Castro HO, Samulski DM, Mello MT. Análise dos sintomas de overtraining durante os períodos de treinamento e recuperação: estudo de caso de uma equipe feminina da Superliga de Voleibol 2003/2004. Rev Bras Med Esporte. 2011;17:397-400.

27. Huffman GR, Park J, Roser-Jones C, Sennet, BJ, Yagnik G, Webner D. Normative SF-36 values in competing NCAA intercollegiate athletes differ from values in the general population. J Bone Joint Surg Am. 2008; 90(3):471-476.

28. Kuehl M, Snyder, A, Erickson SE, McLeod, TV. Impact of prior concussions on health-related quality of life in collegiate athletes. Clin J Sport Med. 2010;20(2):86-91.

29. Brandt R, Liz CM, Crocetta TB, Arab C, Bevilacqua G, Dominski FH, et al. Saúde mental e fatores associados em atletas durante os jogos abertos de Santa Catarina. Rev Bras Med Esporte. 2014;20(4):276-280.

30. Silva EM, Rabelo I, Rubio K. A dor entre atletas de alto rendimento. Rev Bras Psicol Esporte. 2010;3(4):79-97.

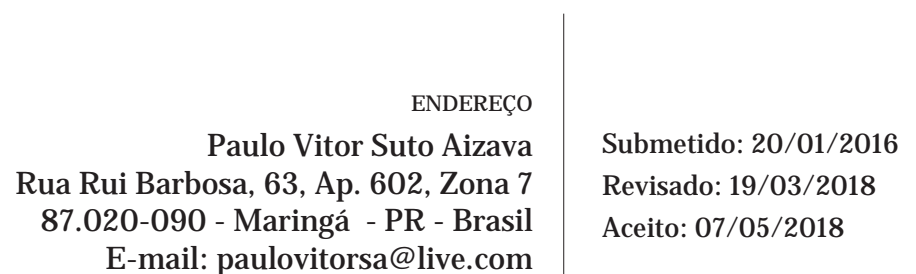

186 • Rev Bras Educ Fís Esporte, (São Paulo) 2021J an-Mar;35(1):177-186 